\title{
Hematoma subaracnoideo espinal tras punción lumbar en paciente con leucemia. Presentación de un caso y revisión de la literatura
}

\author{
J. Ayerbe; D. Quiñones*; E. Prieto** y P. Sousa
}

Servicios de Neurocirugía, Neurorradiología* y Hematología**. Fundación Jiménez Díaz. Madrid.

\section{Resumen}

La compresión mielo-radicular aguda secundaria a un hematoma subaracnoideo espinal (HSAE) es una complicación extremadamente rara tras una punción lumbar (PL). En el mecanismo de producción de estos hematomas han sido implicados diversos factores de riesgo, principalmente la presencia de trastornos de la hemostasia en el paciente.

Presentamos el caso de un varón de 20 años con leucemia aguda y trombocitopenia (26.000 plaquetas/ $\mathbf{m m}^{3}$ ) que, tras ser sometido a una PL, desarrolló una paraparesia con imposibilidad para la bipedestación. La resonancia magnética $(\mathrm{RM})$ demostró la presencia de un hematoma intradural ventral desde D12 hasta L4. Se realizó una laminectomía descompresiva de urgencia y se pudo evacuar parcialmente un hematoma localizado en el espacio subaracnoideo. Al cuarto día postoperatorio, el paciente consiguió caminar sin ayuda, pero falleció un mes después debido a complicaciones sistémicas de su enfermedad.

Sólo se han encontrado 26 casos de HSAE tras PL en la revisión de la literatura que hemos realizado. Se han observado las siguientes características comunes a la mayoría de ellos: asociación con tratamientos anticoagulantes, asociación con trombocitopenia, aparición de síndrome compresivo mielo-radicular de forma retardada, necesidad de tratamiento quirúrgico, buen resultado funcional en la mitad de los pacientes, y corta esperanza de vida en aquellos con enfermedad previa grave. En pacientes leucémicos con tendencia a tener marcada trombocitopenia (quizás por debajo de $\mathbf{2 5 . 0 0 0}$ plaquetas $/ \mathrm{mm}^{3}$ ), el riesgo de desarrollar un HSAE tras PL puede ser elevado.

PALABRAS CLAVE: Hematoma subaracnoideo espinal. Punción lumbar.

Recibido: 15-11-04. Aceptado: 03-12-04 $\overline{\text { Spinal subarachnoid hematoma after lumbar puncture }}$ in a patient with leukemia. Report of a case and review of the literature

Summary

Acute myeloradicular compression due to a spinal subarachnoid hematoma (SSAH) after lumbar puncture (LP) is an extremely rare complication. Several risk factors have been involved in the production of these hematomas, mainly the presence of hemostasis disorders in the patient.

We report the case of a 20 -year-old man with leukemia and thrombocytopenia (26.000 platelets $/ \mathbf{m m}^{3}$ ) who, after undergoing a LP, developed paraparesis and became unable to stand. A magnetic resonance disclosed the presence of a ventral intradural hematoma from D12 to L4. An emergency decompressive laminectomy was performed and an hematoma located in the subarachnoid space was partially removed. On the fourth postoperative day, the patient was able to walk without assistance, but one month later, he died because of systemic complications of his disease.

Only 26 cases of SSAH after LP have been found in the literature review we have performed. In most of them, the following common features have been observed: association with anticoagulant therapies, association with thrombocytopenia, delayed onset of compressive myeloradicular syndrome, need of surgical treatment, good functional outcome in half of patients, and short life expectancy for patients with previous serious illness. Risk for developing a SSAH after LP could be high in leukemia patients with a tendency to have severe thrombocytopenia (perhaps less than 25.000 platelets $/ \mathrm{mm}^{3}$ ).

Abreviaturas. HSAE: hemorragia subaracnoidea espinal. LP: lumbar puncture. PL: punción lumbar. SSAH: spinal subarachnoid hemorrhage. 

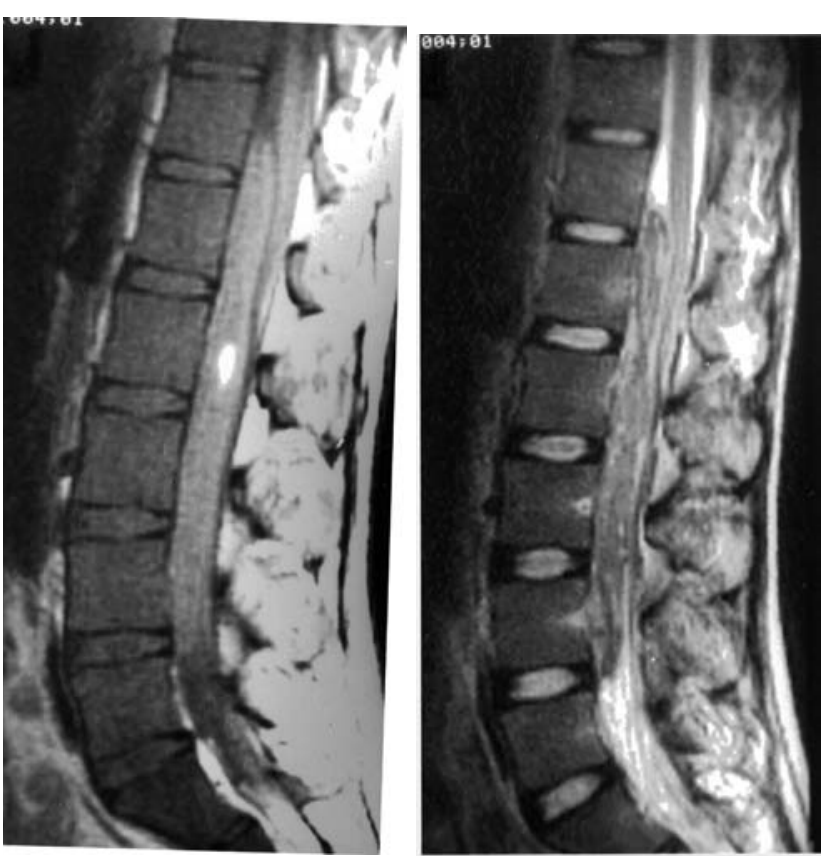

Figura 1. RM sagital dorsolumbar que muestra un proceso expansivo intradural extraneural ventral que comprime el contenido del saco dural desde D12 hasta L4 y que es isointenso con zona central hiperintensa en la secuencia potenciada en T1 (izquierda) y que muestra señal heterogénea en la secuencia potenciada en T2 (derecha).

KEY WORDS: Spinal subarachnoid hematoma. Lumbar puncture.

\section{Introducción}

La PL es una técnica empleada frecuentemente en la práctica médica con fines diagnósticos o terapéuticos, y en los últimos años se está incrementando su utilización en el ámbito de la anestesiología ${ }^{6}$. Una de las conocidas complicaciones de esta técnica, aunque poco habitual, es el desarrollo de hematomas espinales epidurales, subdurales o subaracnoideos, siendo en esta última localización extremadamente raros. En el mecanismo de producción de los mismos se han implicado diversos factores de riesgo observados en el paciente. Entre ellos, los más frecuentes son los trastornos de la hemostasia relacionados con tratamientos anticoagulantes o con determinadas enfermedades. En la mayoría de los casos, el tratamiento de esta complicación ha consistido en la evacuación quirúrgica urgente del hematoma ${ }^{1,8,13,20,21}$. Presentamos el caso de un paciente con leucemia y trombocitopenia que desarrolló un HSAE tras PL, y que fue intervenido quirúrgicamente. Se realiza una revisión de la bibliografía con el objeto de determinar los factores de riesgo más frecuentemente asociados al HSAE, así como las opciones de tratamiento y el pronóstico de los mismos, especialmente en pacientes con leucemia.

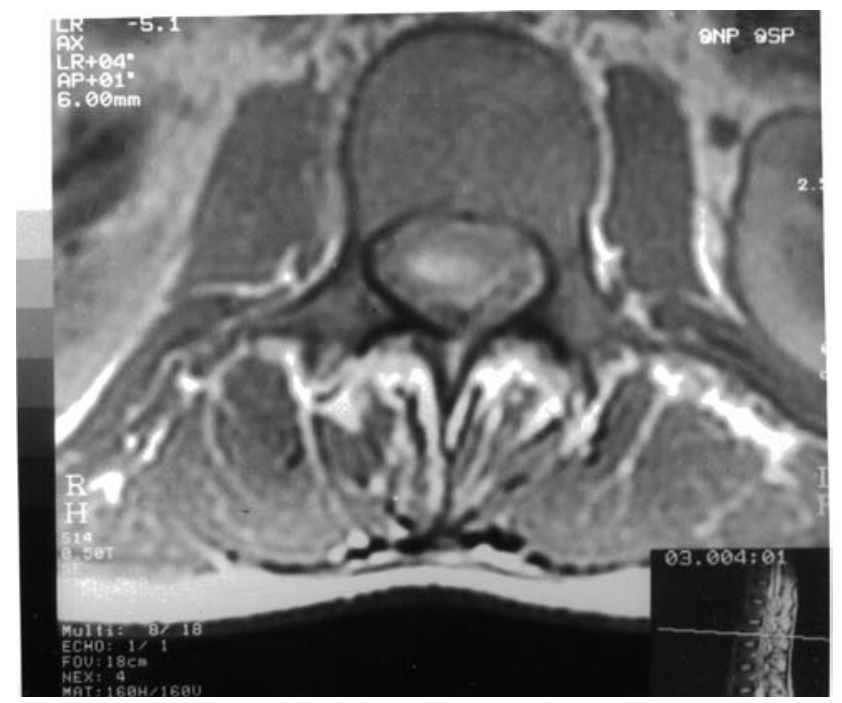

Figura 2. RM axial potenciada en T1 en el nivel L2 que muestra la zona de hiperseñal central con lesión isointensa periférica. Se observan las raices de la cola de caballo desplazadas dorsalmente.

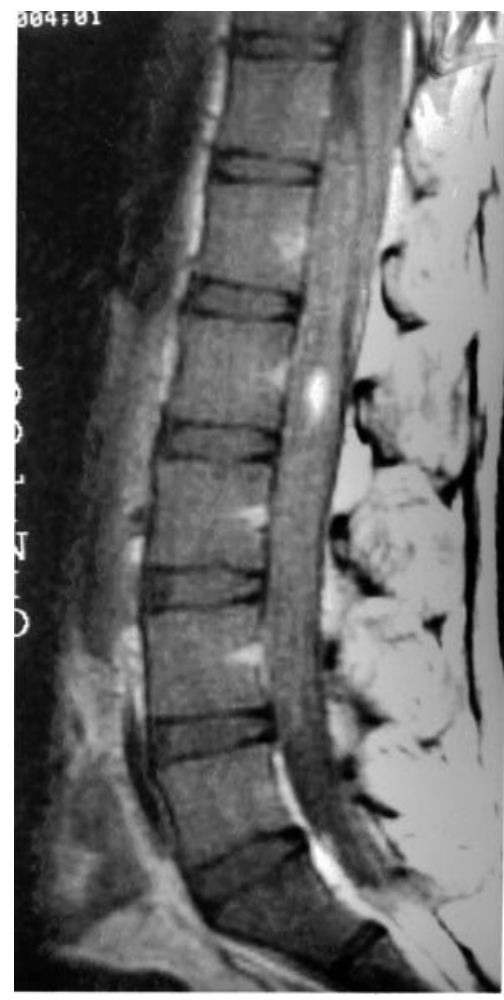

Figura 3. RM sagital dorsolumbar potenciada en T1 tras la administración de Gadolinio intraveno so, en donde no se observan captaciones anómalas de contraste que sugieran la presencia de una lesión subyacente al hematoma.

\section{Caso clínico}

Se trata de un varón de 20 años diagnosticado en Septiembre de 2003 de una leucemia linfoblástica aguda pre-B, al que se administró tratamiento quimioterápico intravenoso de inducción y quimioterapia profiláctica intratecal mediante PL. Tras aplicar varios esquemas de 
tratamiento durante 8 meses, no se consiguió una remisión completa de la enfermedad. En dicho periodo de tiempo se realizaron 4 punciones lumbares para administrar quimioprofilaxis intratecal, sin complicaciones. Los análisis repetidos de líquido cefalorraquídeo (LCR) fueron normales en todas las ocasiones. Los recuentos plaquetarios previos a dichas punciones fueron $34.000,118.000,338.000$ y 161.000 plaquetas $/ \mathrm{mm}^{3}$ respectivamente. Los tiempos de protrombina (TP) y de tromboplastina parcial activada (TTPA) fueron normales, y no se realizó en ningún caso transfusión plaquetaria previa a la PL. En Mayo de 2004, el paciente volvió a ingresar en el hospital para un nuevo ciclo de quimioterapia y programar posteriormente un transplante de médula ósea. Se realizó una nueva PL sin incidencias, pero en esta ocasión se tuvieron que transfundir previamente varios concentrados de plaquetas por tener el paciente una trombocitopenia de $26.000 / \mathrm{mm}^{3}$. En los 2 meses anteriores también había precisado varias transfusiones por la progresiva tendencia a la

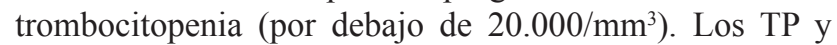
TTPA eran normales. A las pocas horas de esta última PL, el paciente requirió atención por ciatalgia derecha aislada. Al cabo de unas 48 horas, al dolor radicular se asoció pérdida de fuerza en ambos MMII, que evolucionó rápidamente a una paraparesia bilateral, conservando únicamente fuerza motora parcial (3/5) en la flexo-extensión de los pies. No se observaron alteraciones de la sensibilidad ni de los esfínteres. Se practicó de urgencia una RM dorsolumbar (Figuras 1 y 2) que mostró una imagen sugestiva de hematoma ventral intradural extraparenquimatoso que comprimía cono medular y cola de caballo desde D12 hasta L4. Tras administrar gadolinio intravenoso (Figura 3), no se observó captación de contraste sugestiva de lesión subyacente. Posteriormente se realizó una laminectomía descompresiva desde L1 a L4 y una durotomía longitudinal, tras lo que se observó la cola de caballo a gran tensión, contenida por la membrana aracnoidea íntegra. Una pequeña cantidad de coágulo sanguíneo subaracnoideo comprimía dorsalmente el cono medular. La exploración de las zonas laterales demostró la presencia de un gran hematoma ventral contenido también por la membrana aracnoidea. A continuación se realizó una incisión longitudinal de la aracnoides y tras separar las raíces dorsales de la cola de caballo se accedió al coágulo. Sólo fue posible conseguir una extracción subtotal del mismo, ya que estaba parcialmente organizado y adherido a las raíces nerviosas. No se observaron lesiones macroscópicas que pudieran ser el origen del hematoma. Se completó el acto quirúrgico con el cierre del plano dural empleando una plastia liofilizada para ampliación de espacio. La evolución postoperatoria cursó sin complicaciones. El paciente consiguió una recuperación progresiva de la fuerza, pudiendo deambular al cuarto día, sin alteraciones esfinterianas ni dolor radicular y refiriendo solamente un leve acorchamiento residual en miembros inferiores En las semanas sucesivas se continuó con el tratamiento oncológico y el transplante de médula ósea, pero el paciente desarrolló un conjunto de complicaciones sistémicas que ocasionaron finalmente un fracaso multiorgánico y su fallecimiento, transcurrido un mes de la intervención quirúrgica. No se pudo realizar una RM lumbar postquirúrgica debido a la mala situación clínica del paciente.

\section{Discusión}

\section{Generalidades}

La formación de un HSAE que tenga suficiente volumen como para producir un síndrome compresivo medular y/o radicular, es un evento extremadamente raro en la práctica clínica. Esto se explica por diversos motivos, como son la rapidez de dilución de la sangre en el LCR y la pulsatibilidad del mismo, que impedirían la formación del coágulo tras una hemorragia. A estos motivos se añade la propia actividad fibrinolítica del LCR, que además se incrementa en presencia de sangre ${ }^{3,8.20}$.

En la revisión sobre hematomas espinales, realizada por Kreppel y col. ${ }^{13}$, se recogen 613 casos publicados entre 1826 y 1996. Del total de estos casos, los de localización exclusiva o predominantemente subaracnoidea representan el 15,7\% (96 casos). Los factores etiológicos que se pudieron determinar en los pacientes con HSAE fueron, de mayor a menor frecuencia, los siguientes: neoplasias intradurales (en el 30\% de los casos, siendo en su mayoría ependimomas y shwanomas), malformaciones vasculares, trastornos de la hemostasia, y por último, PL/anestesia espinal. En el 9\% de los pacientes no se pudo encontrar una causa determinada. También se describen casos aislados de HSAE en pacientes con traumatismo raquídeo, coartación aórtica, conectivopatías, y vasculitis. Dicho estudio revela que la diferencia más llamativa entre el espectro etiológico de los HSAE y el de los hematomas epidurales/subdurales reside principalmente en el número de casos idiopáticos, que representan hasta un $50 \%$ en este último grupo.

\section{Incidencia}

La incidencia de los HSAE sintomáticos surgidos exclusivamente como complicación de una PL, similares al caso que presentamos, es difícil de estimar. La información disponible parece indicar que es muy baja, de tal modo que, en las revisiones bibliográficas realizadas por diversos autores y actualizada por nosotros mismos, sólo se han podido documentar $26 \operatorname{casos}^{19,13,16,20}$ a los que se tiene que añadir el nuestro. Probablemente, la incidencia sea en realidad más elevada, ya que es difícil contabilizar 
los casos presentados como comunicaciones en congresos, los casos no publicados y los casos en los que, al no realizarse un tratamiento quirúrgico, no se pueda diferenciar con las pruebas de imagen si la localización del hematoma es subdural o subaracnoidea. Algunos autores estiman que la prevalencia general, para todos los tipos de hematoma espinal con síndrome compresivo, sería de 1/200.000 procedimientos de anestesia peridural y 1/320.000 de anestesia subaracnoidea ${ }^{6}$.

\section{Mecanismo de producción y factores asociados}

La mayoría de los autores piensan que los HSAE tras PL están causados por la lesión que la aguja ocasiona en los pequeños vasos sanguíneos que entran en el espacio subaracnoideo acompañando a cada raíz nerviosa. Breuer y col. ${ }^{4}$, en su excelente trabajo sobre este tema, estiman que esta lesión ocurre en más del $25 \%$ de las punciones lumbares, pero consideran que el desarrollo de un hematoma sintomático sólo se ocasionaría en aquellos pacientes con algún trastorno de la hemostasia. En esta red vascular de la cola de caballo, la punción accidental de la vena de Adamkiewicz, que es la de mayor calibre y se suele localizar en torno a las raíces L3 y L4, podría ser el origen de algunos hematomas, especialmente cuando la aguja se dirige muy lateralmente ${ }^{8,13.15}$. La mayor o menor laceración de la aracnoides explicaría la extensión del hematoma al espacio subdural y el que se produzcan hematomas localizados bien de forma aislada en el espacio subdural o subaracnoideo, o bien de forma combinada en ambos espacios ${ }^{20}$.

En $21(77 \%)$ de los 27 casos que hemos revisado, incluido el nuestro, se determinó algún factor asociado que pudo contribuir a la formación del HSAE tras la PL. De este modo, se refiere la existencia de múltiples intentos en la PL en 2 pacientes, de tratamiento anticoagulante/antiagregante previo en 4 , de tratamiento anticoagulante/antiagregante iniciado tras la PL en 7 , y de enfermedades asociadas a trastornos de la hemostasia en 8. Cinco pacientes de este último grupo tenían leucemia y todos presentaban trombocitopenia de mayor o menor cuantía $a^{2.3 .20}$. Aunque la presencia de trombocitopenia en estos pacientes es considerada como factor de riesgo de hemorragia tras una PL, no se ha podido establecer un límite de recuento plaquetario que suponga un incremento de dicho riesgo. Además, en algunas ocasiones, más que por el elemento cuantitativo, las alteraciones de la hemostasia se pueden deber a defectos funcionales de las plaquetas difícilmente objetivables ${ }^{8.21}$. Posiblemente, en los pacientes leucémicos se vean implicados otros factores, todavía mal conocidos, que contribuyan a aumentar el riesgo de HSAE. Apoya esta idea el hecho de que la mayoría de estos pacientes a los que se realiza una PL tienen un mayor o menor grado de trombocitopenia $\mathrm{y}$, sin embargo, no desarrollan esta complicación. No hay guías clínicas basadas en la evidencia que recomienden pautas para la corrección de una trombocitopenia antes de realizar una PL. Wirtz y col. ${ }^{21}$ aconsejan realizar transfusión de plaquetas cuando el recuento es menor de 50.000 plaquetas $/ \mathrm{mm}^{3}$, y Edelson y col. ${ }^{8}$ cuando éste es menor de $20.000 / \mathrm{mm}^{3}$ o cuando éste va descendiendo rápidamente en sucesivos análisis. En nuestro paciente observamos una tendencia al descenso del recuento durante los 2 meses previos a la PL, a pesar de recibir varias transfusiones plaquetarias. Quizás la presencia de este tipo de comportamiento podría añadir un mayor riesgo de HSAE en pacientes con leucemia y debería tenerse en cuenta.

\section{Presentación clínica}

El cuadro clínico suele iniciarse con dolor lumbar agudo tras la PL, con o sin irradiación por territorios radiculares, al que sigue la aparición, tras un periodo variable de tiempo, de un síndrome de compresión medular o de cola de caballo ${ }^{13}$. En muchos pacientes, este síndrome aparece de forma retardada (varios días después de la PL), lo que pudimos observar también en nuestro caso. No hay una teoría satisfactoria que explique esta circunstancia. En este sentido, para Breuer y col. ${ }^{4}$, la lesión de los vasos radiculares (arteriales o venosos) desencadenaría la hemorragia, que continuaría posteriormente con mayor o menor intensidad, en el contexto de una coagulopatía, hasta producir los síntomas. Para Rader ${ }^{17}$, el vaso inicialmente dañado sufriría una segunda rotura favorecida por circunstancias que conlleven aumentos de la presión intraabdominal o intratorácica en el paciente.

\section{Diagnóstico radiológico}

La RM es en la actualidad la prueba de imagen de elección para el diagnóstico de un HSAE. Como ya es conocido, esta técnica ofrece en patología espinal un mayor detalle anatómico y una mayor delimitación de la lesión respecto a otras pruebas. Las características de señal en RM de la sangre en sus diferentes fases de degradación tienen una alta sensibilidad y especificidad para el diagnóstico de hemorragia ${ }^{1314,18}$. Aunque en los casos de hematoma tras PL la relación causa-efecto parezca clara, es aconsejable realizar secuencias de RM con administración de contraste intravenoso para descartar lesiones subyacentes, especialmente en aquellos pacientes con enfermedad neoplásica conocida. Por otro lado, ante la sospecha de una malformación vascular espinal, convendría hacer una angiografía, si bien esta posibilidad no es a veces factible ante una situación de urgencia quirúrgica ${ }^{11}$. La RM delimita muy bien la extensión de un HSAE tras PL, que habitualmente abarca varios niveles vertebrales dorsolumbares, afectando al cono medular y a la cola de caballo. Sin embargo, y a 
pesar de todas las ventajas que tiene esta prueba, ni ésta ni otras exploraciones radiológicas pueden diferenciar en muchos casos si un hematoma intradural espinal es de localización subdural, subaracnoidea o combinada ${ }^{10}$. Esta precisión podría tener cierto interés sobre la planificación y los resultados de un posible tratamiento quirúrgico. En nuestro paciente, el diagnóstico preoperatorio fue de hematoma intradural, sin poder llegar a determinar una localización anatómica más precisa.

\section{Tratamiento y pronóstico}

El tratamiento de los HSAE depende principalmente de las circunstancias clínicas y neurológicas del paciente. $\mathrm{Si}$ el déficit neurológico es significativo o progresivo, y la situación clínica lo permite, el tratamiento de elección consiste en una laminectomía descompresiva y evacuación del hematoma tan pronto como sea posible ${ }^{13,20}$. En el espacio subaracnoideo, el hematoma engloba a las raíces de la cola de caballo, estableciéndose a veces adherencias con las mismas, lo que dificulta en muchas ocasiones su evacuación completa, a pesar de una técnica meticulosa ${ }^{20}$. Si la localización es ventral, como en el caso que presentamos, creemos que se puede optar, para minimizar riesgos, por una evacuación parcial y colocación de una duroplastia para ampliación del espacio. Se han propuesto técnicas mínimamente invasivas como alternativa a la laminectomía, con resultados satisfactorios. De este modo, Schwerdtfeger y col. ${ }^{19}$, logran evacuar un HSAE mediante la inserción de un catéter intratecal y la realización de irrigaciones y succiones repetidas. En pacientes con poca afectación neurológica se puede optar por una actitud expectante junto con un seguimiento clínico-radiológico. En estos casos se han podido comprobar resoluciones espontáneas de los hematomas, con excelentes resultados funcionales

De los 27 pacientes revisados, se realizó laminectomía en 21 (77\%). Para los 19 pacientes en los que se recoge seguimiento, los resultados funcionales fueron buenos en 6 (recuperación completa), regulares en 6 (recuperación parcial) y malos en 7 . Respecto al pronóstico vital, 13 pacientes $(48 \%)$ fallecieron a los pocos días o semanas de la intervención quirúrgica, debido principalmente a complicaciones de su enfermedad previa. En este sentido, el paciente con leucemia que presentamos ilustra una combinación de excelente resultado funcional con un mal pronóstico vital. Este pronóstico global poco satisfactorio, en la serie de casos revisados, contrasta con los mejores resultados obtenidos en pacientes con HSAE de origen indeterminado, según la revisión realizada por Komiyana y col. ${ }^{12}$ En dicho trabajo, 7 de los 9 pacientes estudiados tuvieron una buena recuperación funcional, lo que en parte se explica porque eran pacientes con leve repercusión neurológica previa. En la amplia revisión de Kreppel y col. ${ }^{13}$, que incluye todo tipo de hematomas espinales, el tratamiento quirúrgico se realizó en el $86 \%$ de los casos, y la recuperación funcional completa se obtuvo en el $40 \%$. En dicho estudio se determinaron como factores de peor pronóstico neurológico, para toda la serie en conjunto, el retraso en el tratamiento quirúrgico y la presencia de un síndrome compresivo completo (abolición de funciones motora, sensitiva y esfinteriana). No se encontraron diferencias respecto a la edad del paciente, la rapidez en la progresión de los síntomas, y la extensión del hematoma. Algunos autores han sugerido que la localización ventral de un HSAE es un factor de buen pronóstico $^{12}$, pero esto no ha sido corroborado por otros ${ }^{18}$. Por último, Domenicucci y col. ${ }^{7}$, observan en su serie que los hematomas intradurales con componente subaracnoideo tienen peor pronóstico que los que no lo tienen.

\section{Conclusiones}

Los casos de pacientes con HSAE tras PL referidos en la literatura son escasos, lo que no permite establecer comparaciones significativas con otros tipos de hematomas espinales. En la mayoría de los pacientes con HSAE tras PL se han observado las siguientes características: asociación con tratamientos anticoagulantes/antiagregantes, asociación con trombocitopenia (principalmente en pacientes con leucemia), aparición del síndrome compresivo medular y/o radicular de forma retardada, necesidad de tratamiento quirúrgico, buen resultado funcional en la mitad de los pacientes y corta esperanza de vida en aquellos con enfermedad previa grave. En pacientes leucémicos con tendencia a tener marcada trombocitopenia (quizás por debajo de 25.000 plaquetas $/ \mathrm{mm}^{3}$ ), el riesgo de desarrollar un HSAE tras PL puede ser elevado.

\section{Bibliografía}

1. Adler, M.D., Comi, A.E., Walker, A.R.: Acute hemorrhagic complication of diagnostic lumbar puncture. Pediatr Emerg Care 2001; 17: 184-188

2. Bellamy, E.A., Pérez, D.J., Husband, E.: CT demonstration of a spinal subarachnoid haematoma following lumbar puncture. J Comput Assist Tomogr 1984; 791-792

3. Bladé, J., Gastón, F., Montserrat, E., et al.: Spinal subarachnoid hematoma after lumbar puncture causing reversible paraplegia in acute leukemia. Case report. J Neurosurg 1983; 58: 438-439

4. Breuer, A.C., Tyler, H.R., Marzewski, D.I., Rosenthal, D.S.: Radicular vessels are the most probable source of needle-induced blood in lumbar puncture. Cancer 1982; 49: 2168-2172

5. Cabrera, A., Manchola, 1., Larena, J.A., Martínez de Guereñu, B., Casado, O.: Seguimiento con resonancia magnética de un hematoma medular subaracnoideo ventral espontá- 
neo. Rev Neurol 2001; 32: 1137-1140

6. Castillo, J., Santiveri, X., Escolano, J., Castaño, J.: Hematomas raquídeos con compresión medular relacionados con las anestesias neuroaxiales en España. Rev Esp Anestesiol Reanim 2003; 50: 504-509.

7. Domenicucci, M., Ramieri, A., Ciappetta, P., Delfini, R.: Nontraumatic acute spinal subdural hematoma: report of five cases and review of the literature. J Neurosurg Spine 1999; 91 : $65-73$.

8. Edelson, R.N., Chernik, N.L., Posner, J.B.: Spinal subdural hematoma complicating lumbar puncture: ocurrence in thrombocytopenic patients. Arch Neurol 1974; 31: 134-137

9. Goyal, A., Dua, R., Singh, D., Kumar, S.: Spinal subarachnoid hematoma following lumbar puncture. Neurol India 1999; 47: 339-340.

10. Hausmann, O., Kirsch, E., Radü, E., Mindermann, Th., Gratzl, O.: Coagulopathy induced spinal intradural extramedullary haematoma: report of three cases and review of the literature. Acta Neurochir 2001; 143: 135-140.

11. Kim, Y.H., Cho, K.T., Chung, C.K., Kim, H.J.: Idiopathic spontaneous spinal subarachnoid hemorrhage. Spinal Cord 2004; 42: 545-547.

12. Komiyama, M., Yasui, T., Sumimoto, T., Fu, Y.: Spontaneous spinal subarachnoid hematoma of unknown pathogenesis: case reports. Neurosurgery 1997; 41: 691-694.

13. Kreppel, D., Antoniadis, G., Seeling, W.: Spinal hematoma: a literature survey with metaanalysis of 613 patients. Neurosurg Rev 2003; 26: 1-49.

14. Kulkarni, A.V., Willinsky, R.A., Gray, T., Cusimano, M.: Serial magnetic resonance imaging findings for a spontaneously resolving spinal subdural hematoma: case report
Neurosurgery 1998; 42: 398-400.

15. Masdeu J.C., Breuer A.C., Schoene W.C.: Spinal subarachnoid hematomas: clue to a source of bleeding in traumatic lumbar puncture. Neurology 1979; 29: 872-876.

16. Pai, S.B., Krishna, K.N., Chandrashekar, S.: Post lumbar puncture spinal subarachnoid hematoma causing paraplegia: a short report Neurol India 2002; 50: 367-369.

17. Rader, J.: Chronic subdural hematoma of the spinal cord: report of a case. N Engl J Med 1955; 253: 374-376.

18. Ruelle, A., Zerbi, D., Andrioli, G.: Spinal subarachnoid bleeding of unknown etiology. Case repots. J Neurosurg Sci 2001; 45: 53-57.

19. Schwerdtfeger, K., Caspar, W., Alloussi, S., Strowitzki, M., Loew, F.: Acute spinal intradural extramedullary hematoma: a nonsurgical approach for spinal cord decompression. Neurosurgery 1990; 27: 312-314.

20. Scott, E.W., Cazenave, C.R., Virapongse, C.: Spinal subarachnoid hematoma complicating lumbar puncture: diagnosis and management. Neurosurgery 1989; 25: 287-293.

21. Wirtz, P.W., Bloem, B.R., van der Meer, F.J.M., Brouwer, O.F.: Paraparesis after lumbar puncture in a male with leukemia. Pediatr Neurol 2000; 23: 67-68.

Ayerbe, J.; Quiñones, D.; Prieto, E.; Sousa, P.: Hematoma subaracnoideo espinal tras punción lumbar en paciente con leucemia. Presentación de un caso y revisión de la literarura. Neurocirugía 2005; 16: 447-452.

Correspondencia postal: J. Ayerbe. Servicio de Neurocirugía. Fundación "Jiménez Díaz". Avda. Reyes Católicos, 2. 28040 Madrid. 